\title{
Suicidio y Trastorno Mental
}

\section{Suicide and Mental Disorder}

\author{
Erika Yohanna Bedoya Cardona1 ${ }^{\text {ORCID }}$ - Ludivia Esther Montaño Villalba ${ }^{2}$ ORCID \\ 1,2. Universidad Cooperativa de Colombia \\ Colombia
}

\begin{abstract}
Fecha correspondencia:
Recibido: agosto 20 de 2015.

Aceptado: julio 19 de 2016.
\end{abstract}

Forma de citar:

Bedoya, E., \& Montaño, L. (2016).

Suicidio y Trastorno Mental. Rev.

CES Psicol., 9(2), 179-201.

\section{Open access \\ (c) Copyright \\ Licencia creative commons \\ Etica de publicaciones \\ Revisión por pares \\ Gestión por Open Journal System \\ ISSN: 2011-3080 \\ DOI: http://dx.doi.org/10.21615/ \\ cesp.9.2.12}

Sobre los autores:

1. PhD en Psicología Clínica y

Salud. Docente e Investigadora de

la Facultad de Psicología. Líder del

Grupo de Investigación Familia y

Sociedad. Universidad Cooperativa de Colombia (UCC).

2. MSc en Salud Pública. Docente de Cátedra Programa de Psicología. (UCC).

\section{Comparte}

\section{G.898 $\mathbb{R}^{\circ}$ 因}

\section{Resumen}

La Organización Mundial de la Salud define el suicidio como el acto deliberado de quitarse la vida, causándose una lesión, con un grado variable en la intención de morir. El suicidio es la cuarta forma de muerte violenta en Colombia (Cifuentes, 2013). En el 2014 se reportaron 4,33 casos por 100.000 habitantes, siendo los grupos etarios de 20 a 24 y de 70 a 74 años los que presentaron mayores tasas a nivel nacional (Ramírez \& Naranjo, 2014). La literatura reporta que en el $90 \%$ de casos de suicidio se encuentra algún trastorno mental asociado, lo que se constituye en un problema de salud pública. Este artículo de revisión se propone describir la relación entre trastornos mentales y conducta suicida, con el fin de contribuir al entendimiento del fenómeno y resaltar la necesidad de crear e implementar estrategias de atención temprana.

Palabras Clave: Suicidio, Ideación Suicida, Salud Mental, Salud Pública, Psicopatología, Trastorno Mental.

\section{Abstract}

The World Health Organization defines suicide as the act of deliberately killing oneself, causing injury or damage, with varying degrees of intention to die. Suicide is the fourth form of violent death in Colombia. In 2014 , 4.33 cases per 100,000 inhabitants were reported; the target age groups, with the higher rates nationwide, consisted of 20-24 and 70-74 years old. Papers reported that $90 \%$ of suicides are associated with mental disorder therefore, it should be considered a public health problem. This study aims to review scientific literature to describe the relationship between mental disorders and suicidal behavior, highlighting the need to establish and implement strategies for early intervention in populations at suicide risk.

Keywords: Suicide, Suicidal Ideation, Mental Disorders, Mental Health, Public Health, Psychopathology.

\section{Introducción}

La Organización Mundial de la Salud (OMS) define el suicidio como "el acto deliberado de quitarse la vida" (2012a, p. 75). La conducta suicida se puede expresar como un continuo que va desde la ideación, planeación y tentativa hasta el suicidio consumado (Ministerio de Salud y Protección Social, 2014). Desde 1970 la OMS identificó el acto suicida como un problema de salud pública, y en el 2013 realizó un estudio epidemiológico encontrando que en el mundo 9.000 personas intentan suicidarse diariamente y cada año se cometen alrededor de un millón de suicidios; esto significa una 
Pág 180

En el año 2013 se expidió la Ley 1616 de Salud Mental, mediante la cual se busca garantizar el ejercicio del derecho a la salud mental, se establecen los reglamentos para la promoción y prestación de servicios integrales. En el 2014 se publicó el Documento Propuesta de Ajuste de la Política Nacional de Salud Mental para Colombia (Ministerio de Salud y Protección Social -Organización Panamericana de la Salud-OPS-/OMS, 2014) muerte cada 40 segundos, ubicando al suicidio entre las tres primeras causas de muerte en personas de 15 a 44 años (M, 2013). Se estima además que para el año 2020, aproximadamente 1,53 millones de personas morirán por suicidio, lo que representa un promedio de una muerte cada 20 segundos y un intento cada 1-2 segundos (Bertolote \& Fleischmann, 2002).

Como un intento de consensuar medidas preventivas, en el año 2012 la OMS creó el Programa de Prevención del Suicidio (SUPRE) (2012b). A este esfuerzo se han unido algunos países a nivel latinoamericano y mundial, realizando diversos estudios y propuestas de abordaje como, por ejemplo, la Asociación Internacional del Teléfono de la Esperanza (ASITES), cuya misión es promover la salud emocional de las personas en crisis mediante redes de ayuda preventiva de carácter gratuito.

Hace casi 20 años existe en Colombia una Política de Salud Mental Ministerio de Salud República de Colombia, 1998, Resolución $N^{\circ}$ 2358) que dicta las normas científicas, técnicas y administrativas reguladoras de la calidad de servicios para esta área de la salud, y se han ido mejorando las condiciones a través de propuestas como los Lineamientos de Política de Salud Mental para Colombia del Ministerio de la Protección Social y la Fundación FES Social (2005), el Modelo de Gestión Operativa para el Componente de Salud Mental en Atención Primaria en Salud (Rey Sarmiento, 2009) y el Observatorio Nacional de Salud Mental (Ministerio de Salud y Protección Social República de Colombia, 2011).

En el año 2013 se expidió la Ley 1616 de Salud Mental, () mediante la cual se busca garantizar el ejercicio del derecho a la salud mental, se establecen los reglamentos para la promoción y prestación de servicios integrales. En el 2014 se publicó el Documento Propuesta de Ajuste de la Política Nacional de Salud Mental para Colombia Ministerio de Salud y Protección Social -Organización Panamericana de la Salud -OPS-/OMS, 2014). Ese año el mismo Ministerio reconoció importantes restricciones en la aplicación y resultados del Sistema de Salud de Colombia, resaltando que la atención en salud mental es limitada en su manejo oportuno y temprano de las necesidades de la población, presenta problemas de calidad y está basada en un modelo hospitalario de prestación de servicios, y propone el Programa Nacional de Salud y Medicina Familiar y Comunitaria para fortalecer la formación, competencias e interdisciplinariedad de los diferentes profesionales de la salud.

En el año 2015, la cuarta Encuesta Nacional de Salud Mental en el país, desarrollada por el Ministerio de Salud y Protección Social, COLCIENCIAS, la Pontificia Universidad Javeriana y Datos, Procesos y Tecnología S.A.S., presentó datos epidemiológicos relacionados con la percepción subjetiva de bienestar y relaciones interpersonales, apoyo social, estrés, violencia, etc., con el fin de que fueran tenidos en cuenta en los planes de promoción de la salud mental y prevención oportuna de problemas y trastornos mentales, por ejemplo, mediante estrategias de fortalecimiento de la empatía, reconocimiento y participación social. En enero del 2016 se publicó la Política de Atención Integral en Salud ( $( \pm)$ para asegurar el acceso a servicios fundamentales a través del mejoramiento de la calidad de los mismos, fortaleciendo la infraestructura hospitalaria, la sostenibilidad financiera, las estrategias preventivas con enfoque familiar, comunitario e intercultural, el talento humano y la incorporación de Telesalud (actividades de promoción, prevención, diagnóstico, tratamiento y rehabilitación mediante la utilización de tecnologías de información y comunicación, para facilitar el acceso de población que vive en lugares remotos). 
Este artículo se propone describir la estrecha relación entre trastornos mentales y la conducta suicida, con el fin de contribuir al entendimiento del fenómeno y justificar la urgencia de reconocer y actuar no sólo mediante tratamientos e intervenciones sobre las dificultades y patologías, sino también a través de planes y actividades de prevención y atención temprana como uno de los principales objetivos de la salud pública..
En Colombia, el suicidio es la cuarta forma de muerte violenta' (Cifuentes, 2013). En 2014 se reportaron 4,33 casos por 100.000 habitantes, siendo los grupos etarios de 20 a 24 y de 70 a 74 años los de mayores tasas $(6,50)$ a nivel nacional. El grupo con más casos es el de hombres entre 20 y 29 años seguido por el de mujeres entre 15 y 17 años. Algunos factores psicosociales asociados al suicidio son duelos, dificultades familiares o de pareja, desempleo, jubilación, divorcio o viudez, diagnósticos de enfermedades graves, trastorno mental, abuso de sustancias psicoactivas y alcohol (Ramírez \& Naranjo, 2014).

Las consecuencias son devastadoras tanto para las personas que realizan actos suicidas y sus familiares como para el Estado, debido a las lesiones físicas, traumas emocionales y mentales, hospitalizaciones y alta inversión que implica un tratamiento para la recuperación integral. Además, el suicidio genera un impacto económico debido a la pérdida de población en edades productivas. En Colombia se estima que, en el año 2006, los años perdidos a causa del suicidio fueron 57.078 por año, de los cuales 19.590 correspondían a personas en edades entre 18 y 24 años (Rodríguez, 2007).

Por otra parte, las personas con trastornos mentales constituyen una población con alto grado de afectaciones (De Hert et al., 2011), entre ellas el suicidio. Los trastornos mentales son un importante factor de riesgo, ya que cerca del $90 \%$ de personas que cometen suicidio presentan un diagnóstico psiquiátrico (Phillips, 2010; Windfuhr \& Kapur, 2011) y cuando éstos se agravan suele presentarse mayor mortalidad por suicidio, según reportan autopsias psicológicas (Cavanagh, Carson, Sharpe, \& Lawrie, 2003; Saha, Chant, \& McGrath, 2007). El suicidio es, entonces, superior en dicha población, lo que representa un desenlace muy costoso para las familias y la sociedad (Ajetunmobi, Taylor, Stockton, \& Wood, 2013; Whiteford et al., 2013).

Es común que las personas con este tipo de diagnósticos asistan a servicios de atención primaria (Cohen, 2006) y consulten profesionales de salud durante el año previo al suicidio (Borges et al., 2005; Espinosa, Blum Grynberg, \& Romero Mendoza, 2009; Hunt et al., 2006a; Luoma, Martin, \& Pearson, 2002), y la cantidad de necesidades no satisfechas en esta población aumentan la carga social, el costo mismo de la enfermedad y las pérdidas humanas (Wittchenet al., 2011). Existe en la población con trastornos mentales un riesgo de suicidio relacionado con el alta hospitalaria tras una crisis propia del trastorno o un intento de suicidio: se ha reportado que el periodo de mayor riesgo son las cuatro primeras semanas después del alta (Goldacre, Seagroatt, \& Hawton, 1993), lo que podría explicarse por una percepción de pérdida de apoyo, menor supervisión, recaída al verse nuevamente expuestos a problemas en el medio habitual, abandono del tratamiento, o que simplemente no estén del todo recuperados, con lo cual se justifica la importancia del mantenimiento del tratamiento con medicación profiláctica en personas con episodios recurrentes, adecuado apoyo comunitario, atención especial a los signos precoces de recaída y diseño de planes que mitiguen el riesgo.

Teniendo en cuenta este panorama general, este artículo se propone describir la estrecha relación entre trastornos mentales y la conducta suicida, con el fin de contribuir al entendimiento del fenómeno y justificar la urgencia de reconocer y actuar no sólo mediante tratamientos e intervenciones sobre las dificultades y patologías, sino también a través de planes y actividades de prevención y atención temprana como uno de los principales objetivos de la salud pública (OMS, 2013; Pérez, 2011).

1 Según la Psiquiatría Forense el suicidio es entendido como una forma de muerte violenta (las otras dos son el homicidio y los accidentes), ya que se atribuye a causas externas a la persona y se da a través de "mecanismos traumáticos o fuerzas extrañas que irrumpen violentamente en el organismo" (Borges Gonzáles, Vidal Palmer, \& Pérez Milan, 2006, p. 1) 
Pág 182

Durante más de 50 años Colombia ha padecido múltiples conflictos sociales y políticos generando desplazamientos forzados, secuestros, torturas, masacres, desapariciones, homicidios, violencia intrafamiliar y de género, maltratos, abusos sexuales, explotación, abuso de alcohol y sustancias psicoactivas, etc. (Centro Nacional de Memoria Histórica, 2013), desencadenando situaciones de estrés, comportamientos autodestructivos (Cervantes \& Melo Hernández, 2008) y trastornos mentales.
La presente revisión se realizó mediante una búsqueda bibliográfica de artículos, libros, políticas y leyes, utilizando los términos tomados de la Biblioteca virtual en salud DeCS (Descriptores en Ciencias de la Salud) y del Tesauro MeSH (Medical Sucject Headings): "suicidio, ideación suicida, espectro suicida, enfermedad mental, trastorno mental, psicopatología, suicidio y trastorno mental, suicidio y psicopatología, suicidio y enfermedad mental, factores de riesgo, salud pública"; en las siguientes bases de datos: Science Direct, PubMed, Ovid, Scielo, Psicodoc y Google Académico.

\section{Implicaciones en el Contexto Colombiano}

Según la OPS (2003), trastornos mentales como la depresión, trastornos por consumo de alcohol y abuso de sustancias, la violencia, las guerras, los desastres, la aculturación² (de pueblos indígenas o personas desplazadas, entre otros), la discriminación, el aislamiento, las pérdidas y diversos entornos sociales, constituyen factores de riesgo de suicidio, así como las dificultades para acceder a la atención en salud, la disponibilidad de los recursos para suicidarse y el sensacionalismo de algunos medios masivos de comunicación cuando informan sobre los casos de suicidio ocurridos.

Durante más de 50 años Colombia ha padecido múltiples conflictos sociales y políticos generando desplazamientos forzados, secuestros, torturas, masacres, desapariciones, homicidios, violencia intrafamiliar y de género, maltratos, abusos sexuales, explotación, abuso de alcohol y sustancias psicoactivas, etc. (Centro Nacional de Memoria Histórica, 2013), desencadenando situaciones de estrés, comportamientos autodestructivos (Cervantes \& Melo Hernández, 2008) y trastornos mentales. Entre las causas directas de los trastornos mentales se encuentran los problemas psicosociales y la descomposición del núcleo familiar, y entre las indirectas el deterioro de la situación social y económica de la población, así como el bajo nivel de calidad de vida. Por otro lado, algunos efectos directos de los trastornos mentales son el aumento de costos de promoción, prevención y tratamiento, y la disminución de la capacidad laboral y productiva; y entre los indirectos se encuentran el aumento de casos de violencia intrafamiliar, violencia sexual y de género, elevado consumo de sustancias psicoactivas, deterioro social y económico (Proyecto de Ordenanza No 040 por medio de la cual se establecen los Lineamientos de la Política Pública en Salud Mental y Convivencia Social del Departamento de Santander, 2014). Todos estos fenómenos, como se puede observar, constituyen un círculo vicioso en el que se mantienen y aumentan las afectaciones y consecuencias.

\section{Asociación entre Suicidio y Trastorno Mental}

Los trastornos mentales más frecuentes en adultos que han realizado intentos de suicidio son depresión, distimia, bipolaridad, trastorno de personalidad, ansiedad, agorafobia, abuso de sustancias (drogas psicoactivas, alcohol y tabaco), esquizofrenia, somatización y trastornos alimentarios como la anorexia nerviosa (Balhara \& Verma, 2012; Chesney, Goodwin, \& Fazel, 2014; Rodríguez \& Guerrero, 2005; Silva, Vicente, Saldivia, \& Kohn, 2013; Soloff, Fabio, Kelly, Malone, \& Mann, 2005). En adolescentes se encuentran trastornos de conducta, depresión, fobias simples y ansiedad generalizada (Pelkonen \& Marttunen, 2003). También existe relación entre suicidio y trastornos de alimentación en adolescentes mujeres, trastornos de conducta en hombres (límite y sociópata) y abuso de sustancias en ambos sexos (Bhatia \& Bhatia, 2007).

2 Según la Real Academia Española [RAE] (2014), la aculturación consiste en "incorporar a un individuo o a un grupo humano elementos culturales de otro grupo" que, en el caso colombiano, se ha presentado en gran medida en el fenómeno del sometimiento de cientos de personas víctimas de desplazamiento forzado debido al conflicto armado, y que han sido obligadas a perder sus valores culturales para tener que adaptarse a otros. 
La depresión puede incrementar hasta 12 veces el riesgo de suicidio cuando predomina la desesperanza, asociada con intencionalidad y alta letalidad (Aliaga, Rodríguez, Ponce, Fristancho, \& Vereau, 2006).
Existe mayor riesgo de suicidio en los primeros meses después de ser diagnosticado con algún trastorno afectivo (Castro-Díazet al., 2013), depresión psicótica y trastorno bipolar (Steele \& Doey, 2007). El riesgo aumenta en los primeros días o semanas de inicio de un tratamiento farmacológico ya que en dicho período suele empeorar la sintomatología (Healy \& Alfred, 2005; Perlis et al., 2007), y en los días siguientes al alta hospitalaria, sobre todo en aquellos que consultan en urgencias (Gairin, House, \& Owens, 2003; Hansagi, Olsson, Hussain, \& Onlén, 2008), reportando un riesgo 100 veces mayor $(H o, 2003)$ entre los tres días y la semana después de salir del hospital (Hunt et al., 2009).

También se ha encontrado evidencia de que a mayor número de trastornos mentales comórbidos es mayor el riesgo de suicidio, siendo los que se anotan a continuación los principales:

Depresión. Según el Manual Diagnóstico y Estadístico de los Trastornos Mentales [DSM-V] (American Psychiatric Association - APA, 2013), la depresión es un período en el que se presenta un cambio del funcionamiento previo, estado de ánimo decaído, pérdida de interés o placer, cambios de apetito, sueño, actividad motora, sentimiento de inutilidad o culpa, dificultad para concentrarse y "pensamientos de muerte recurrentes (no sólo miedo a morir), ideas suicidas recurrentes sin un plan determinado, intento de suicidio o un plan específico para llevarlo a cabo" (p. 105).

A nivel sintomatológico existe una relación entre depresión y suicidio (Aristizábal, González, Palacio, García, \& López, 2009; Bolton, Belik, Enns, Cox, \& Sareen, 2008; González \& Camejo, 2014; Harwitz \& Ravizza, 2000; Nock et al., 2008; Palacio et al., 2007). Se estima que la depresión por sí sola aumenta el riesgo de suicidio (Kessler, Berglund, Borges, Nock, \& Wang, 2005); al respecto Hagnell, Lanke, Rorsman y Ojesjo (1982, citados por Hernández, González-Elias, \& López, 2013) sostienen que las personas deprimidas presentan tasas de suicidio 44 veces superiores a las personas sin depresión, y 8 veces superiores al resto de los pacientes psiquiátricos. En la tentativa de suicidio, la depresión juega un papel determinante y afecta principalmente a los adolescentes (Cabra, Infante, \& Sossa, 2010; Toro, Paniagua, González, \& Montoya, 2009).

En personas con trastorno depresivo al menos un $27 \%$ han tenido un intento de suicidio a lo largo de sus vidas (Nemeroff, Compton, \& Berger, 2001). La depresión puede incrementar hasta 12 veces el riesgo de suicidio cuando predomina la desesperanza, asociada con intencionalidad y alta letalidad (Aliaga, Rodríguez, Ponce, Fristancho, \& Vereau, 2006). La vivencia de sufrimiento en la depresión, evidente en cartas de personas que han realizado actos suicidas, se ha relacionado con mayor frecuencia e intensidad de ideación y conductas suicidas (Olié, Guillaume, Jaussent, Courtet, \& Jollant, 2010).

Ansiedad. Según el DSM-V (APA, 2013) los trastornos de ansiedad "se caracterizan por miedo excesivo ante amenazas reales o percibidas y ansiedad como anticipación de amenazas futuras. Se ha encontrado asociación de varios trastornos de ansiedad con ideación e intentos suicidas" (p. 193), y las personas con fobias específicas "tienen un $60 \%$ más de probabilidad de intentos de suicidio que personas sin el diagnóstico. Sin embargo, esta elevada tasa puede deberse a la comorbilidad con trastornos de personalidad y otros trastornos de ansiedad" (p. 201). También se ha hallado que "la presencia de ataques de pánico y diagnóstico de trastorno de pánico en los últimos 12 meses están relacionados con una mayor tasa de intentos e 
Pág 184

En un estudio realizado por Baca y Aroca (2014) se encontró que quienes presentaban ansiedad y depresión eran más impulsivos, realizaron más intentos suicidas, y tenían antecedentes familiares de conducta suicida, abuso sexual y emocional en la infancia. ideación suicida, incluso cuando se tienen en cuenta la comorbilidad, una historia de abuso infantil y otros factores de riesgo de suicidio" (p. 212).

Otros estudios han mostrado que el trastorno de ansiedad aumenta el riesgo de ideación e intento suicida (Bartels et al., 2002; Norton, Temple, \& Pettit, 2008; Sareen et al, 2005). Casi el $20 \%$ de los pacientes con un trastorno de crisis de ansiedad y fobia social hacen intentos de suicidio infructuosos (Caycedo et al., 2010). Se consideran factores de riesgo de suicidio los trastornos de ansiedad moderadamente severos, reacciones transitorias de ajuste, ansiedad como rasgo de personalidad y características obsesivas (Chioqueta \& Stiles, 2003; Joiner, Brown, \& Wingate, 2005), por lo que la valoración de la gravedad de la ansiedad ayudaría a identificar pacientes en riesgo de suicidio (Sharma, 2003).

En un estudio realizado por Baca y Aroca (2014) se encontró que quienes presentaban ansiedad y depresión eran más impulsivos, realizaron más intentos suicidas, y tenían antecedentes familiares de conducta suicida, abuso sexual y emocional en la infancia.

Trastorno Bipolar. Según el DSM-V (APA, 2013), el trastorno bipolar implica episodios maníacos, seguidos o antecedidos por episodios hipomaníacos o de depresión mayor. Entre el $10 \%$ y $15 \%$ de personas con trastorno bipolar consuman el suicidio, usualmente al inicio de la enfermedad (Goodwin \& Jaminson, 2007) y en las fases depresivas (Post, 2005). Strakowski, McElroy, Keck y West (1996) identificaron en pacientes con trastorno bipolar hasta un $90 \%$ de tendencia al suicidio, y altas puntuaciones en una escala de depresión como el factor más agravante; además se ha establecido que el riesgo de suicidio es 22 veces mayor en pacientes diagnosticados con trastorno bipolar que en población general (Tondo, Hennen, \& Baldessarini, 2001).

Abuso de sustancias psicoativas. Según el DSM-V (APA, 2013) las condiciones clasificadas como inducidas por uso o abuso de sustancias son: "intoxicación, abstinencia y otros trastornos (psicóticos, bipolar y relacionados, depresivos, de ansiedad, obsesivo-compulsivos y relacionados, del sueño, disfunciones sexuales, delirio, y neurocognitivos)" (p. 481). En general, el riesgo para todos los trastornos relacionados con sustancias se asocia con sobredosis accidentales y deliberadas. "La repetida intoxicación y abstinencia pueden estar asociadas con depresiones severas lo suficientemente intensas como para dar lugar a intentos y suicidios. Sin embargo, los datos disponibles sugieren que no deben confundirse sobredosis accidentales no fatales con intentos de suicidio" (p. 544).

El abuso de sustancias presenta altos niveles de comorbilidad con otros trastornos mentales (Davis, Uezato, Newell, \& Frazier, 2008; Dougherty, Mathias, Marsh, Moeller, \& Swann, 2004; Pan et al., 2012; Park, Scheep, Jang, \& Koo, 2006), aumentando su incidencia de un 19\% a 45\% (Bakken \& Vaglum, 2007). Cerca del 50\% de quienes se suicidan están intoxicados y el $18 \%$ de las personas con diagnóstico de alcoholismo se suicidan (Maris, 2002).

El abuso de alcohol y sustancias psicoactivas relacionado con muertes por suicidio fluctúa entre un $5 \%$ y un $27 \%$, y el riesgo de suicidio para las personas con diagnóstico de alcoholismo a lo largo de su vida se sitúa alrededor del 15\% (Miles, 1997). El abuso de sustancias y alcohol es un importante predictor de suicidio (Ocampo, Bojorquez, \& Cortés, 2009) y depresión (Lecrubier, 2002). La frecuente comorbilidad 
Algunos autores explican la asociación entre consumo de sustancias y suicidio (Bernal et al., 2007; Borges et al., 2000) proponiendo que la intoxicación puede reducir la inhibición y aumentar el riesgo de un acto de suicidio impulsivo, alterar la capacidad de juicio y potenciar la disforia (Santamarina et al., 2004), generar disfunción cerebral, cambios neuropsicológicos, cambio en estado de ánimo y conducta violenta, que son potenciados cuando se consume más de una sustancia (Conner, Beautrias, \& Conwell, 2003). entre trastorno depresivo y consumo de sustancias es casi tres veces más alta que en población general (Agosti \& Levin, 2006), y en el caso de dependencia de alcohol hay hasta dos tercios más de asociación (Beghi, Rosenbaum, Cerri, \& Cornaggia, 2013; Santamarina, Iglesias, \& Alonso, 2004).

El riesgo de suicidio en personas que consumen sustancias aumenta 2.6 veces. El alcohol (72,9\%), el cannabis y los sedantes (6,8\%), los estimulantes (9,6\%), los opioides (Borges, Walter, \& Kessler, 2000) y los fármacos de prescripción son los más utilizados para llevar a cabo actos suicidas (Agosti \& Levin, 2006; Preuss et al., 2002). También, el abuso/dependencia de cocaína tiene una prevalencia de 40,5\% en casos de ideación suicida y 39, 2\% de intentos (Garlow, Purselle, \& D'Orio, 2003).

Algunos autores explican la asociación entre consumo de sustancias y suicidio (Bernal et al., 2007; Borges et al., 2000) proponiendo que la intoxicación puede reducir la inhibición y aumentar el riesgo de un acto de suicidio impulsivo, alterar la capacidad de juicio y potenciar la disforia (Santamarina et al., 2004), generar disfunción cerebral, cambios neuropsicológicos, cambio en estado de ánimo y conducta violenta, que son potenciados cuando se consume más de una sustancia (Conner, Beautrias, \& Conwell, 2003).

Trastornos de personalidad. Definidos por el DSM-V (APA, 2013) como un patrón perdurable e inflexible "de experiencia interna y comportamiento que se desvía notablemente de las expectativas de la cultura del individuo, y se manifiesta en la cognición, afectividad, funcionamiento interpersonal y control de impulsos" (p. 359). Estos trastornos se presentan hasta en un 70\% en personas suicidas (Mejía, Sanhueza, \& González, 2011), y se encuentran implicados rasgos como autoestima pobre, impulsividad, ira y agresividad (Guo \& Harstall, 2002), siendo las dimensiones de desregulación emocional y la impulsividad las que mayor prevalencia han presentado en estudios retrospectivos (Frías Ibañez, Vázquez Costa, del Real Peña, \& Sánchez del Castillo, 2012).

Las personas que padecen trastornos de personalidad tienen mayor riesgo de suicidio (Echávarri et al., 2015; Matschnig. Fruhwald, \& Frottier, 2006). Hay especial evidencia para los trastornos límite de personalidad (Samuels, Nestadt, Romanoski, Folstein, \& McHugh, 1994), narcisista e histriónico (Mardomingo, 2000), antisocial (Verona, Patrick, \& Joiner, 2001) y esquizotípico (Sollof, Lynch, \& Kelly, 2002). El 56\% de personas que se suicidan presentan dichos trastornos en comorbilidad con abuso de drogas y alcohol (He, Felthous, Holzer, Nathan, \&Veasey, 2001), al igual que el $65 \%$ de quienes lo intentan (Arbanas, Bicanic, Grba-Bujevic, \& Mlinac-Lucijanic, 2006; Lejoyeux \& Marinescu, 2006).

El intento de suicidio se considera un criterio diagnóstico de trastorno de la personalidad tipo límite (TPL) (LeGris \& van Reekum, 2006; Yoshida et al., 2006). En personas con dicho trastorno se ha encontrado una prevalencia de 60\% a $80 \%$ de comportamientos auto lesivos (Mendieta, 1997) y un 10\% de suicidios consumados en menores de 30 años (Soloff et al., 2002), en su mayoría mujeres (Qin, 2011), ya que en el TPL existe una fuerte presencia de inestabilidad emocional (Yen et al., 2004) conductas autodestructivas e impulsivas (Hawton \& Van Heeringen, 2009; Swann, Lijffijt, Lane, Steinberg, \& Moeller, 2009) como conducción temeraria, gastos, robos, atracones y purgas, abuso de sustancias, encuentros sexuales sin protección, auto mutilaciones e intentos suicidas (APA, 2001; Sodeberg, 2001). En el estudio de Espinosa et al. (2009) se encontró que el $46 \%$ de los pacientes con TPL obtuvo puntua- 
Pág 186

Los pacientes con diagnóstico de esquizofrenia que presentan aislamiento social y familiar, falta de apoyo y dificultades económicas tienden a cometer más suicidios (Labonté et al., 2013; Labonté et al., 2012; Pavez, Santander, Carranza, \& Vera-Villaroel, 2009), al igual que los más jóvenes, de sexo masculino, con historia de suicidios en la familia y abuso de sustancias (Hunt et al., 2006b). ciones elevadas en una escala de desesperanza, asociada con síntomas de ansiedad crónica e incertidumbre frente al futuro.

Esquizofrenia. En el DSM-V (APA, 2013) se define la esquizofrenia como una "alteración con síntomas como delirios, alucinaciones, discurso y comportamiento desorganizados, y síntomas negativos" (p.54). Y el DSM-IV (APA, 1995) señalaba que "aproximadamente el $10 \%$ de los sujetos con esquizofrenia se suicidan" (p.286). Ciertos estudios han centrado su atención en la relación entre conducta suicida y esquizofrenia (Addington, 2006; Hoang, Stewart, \& Goldacre, 2011; Lecrubier, 2002). En los primeros años de la enfermedad, entre un $5 \%$ y $15 \%$ de pacientes mueren por suicidio (Hor \& Taylor, 2010), y éste usualmente tiene más que ver con síntomas depresivos, ya que la comorbilidad entre depresión y esquizofrenia tiene una prevalencia de 17\% a 65\% (Carpenter \& Buchanan, 1993). La depresión postpsicótica está presente en más de un $25 \%$ de pacientes (Meltzer, 1998), incrementando el riesgo de recaídas, empeorando la respuesta al tratamiento y generando mayor deterioro funcional (Hausmann \& Wolfgang, 2000).

Los pacientes con diagnóstico de esquizofrenia que presentan aislamiento social y familiar, falta de apoyo y dificultades económicas tienden a cometer más suicidios (Labonté et al., 2013; Labonté et al., 2012; Pavez, Santander, Carranza, \& Vera-Villaroel, 2009), al igual que los más jóvenes, de sexo masculino, con historia de suicidios en la familia y abuso de sustancias (Hunt et al., 2006b). Usualmente se ven afectados por estados de desesperanza y desesperación, frecuentes recaídas, sintomatología grave, pobre funcionamiento social, deterioro mental, poca adherencia al tratamiento o excesiva dependencia del mismo (Harkavy-Friedmanet al., 1999). Tal y como reporta Williams (1992), el 40\% de personas con esquizofrenia que se suicidan presentaban impulsividad, depresión, tensión y estrés grave y constante, mayor consciencia de la enfermedad y sus consecuencias, presentando el denominado síndrome lineal de consciencia de la enfermedad (Carpenter \& Buchanan, 1993) caracterizado por un ciclo de desmoralización - depresión - suicidio, ya que, evidenciar las consecuencias, limitaciones y el deterioro en la calidad de vida que conlleva la enfermedad (Abela \& Seligman, 2000), propicia un alto riesgo de suicidio (Schwartz, 2000).

\section{Factores y Modelos Explicativos.}

Según Rascón et al. (2004), un trastorno mental por sí solo no puede explicar la conducta suicida; así mismo, en la literatura se encuentra amplia evidencia explicativa de la asociación existente entre el suicidio y diversas variables, como se describe a continuación.

En la relación entre funcionamiento familiar y conducta suicida se resaltan aspectos como las dificultades interpersonales (Cantoral \& Betancourt, 2011), comunicación conflictiva (Andrade, 2012; Espinoza-Gómez, Zepeda-Pamplona, Hernández-Suárez, Newton-Sánchez, \& Plasencia-García, 2010), pobre cercanía afectiva (Muñoz, Pinto, Callata, Napa, \& Perales, 2005), altos niveles de control parental, estructura familiar inestable, historia familiar de suicidio, violencia intrafamiliar, abuso sexual en la infancia (González-Quiñones \& De la Hoz Restrepo, 2010), maltrato físico y psicológico (Hernández et al., 2013), negligencia, crianza en hogares monoparentales (Guibert Reyes \& Niurka Torres, 2001; Salirrosas-Alegría \& Saavedra-Castillo, 2014), etc., los cuales limitan la activa y adecuada participación de todos sus miembros y dificultan la satisfacción de necesidades básicas (Valadez-Figueroa, Amezcua-Fernández. Quintanilla-Montoya, \& González-Gallegos, 2005; Guibert Reyes \& Niurka Torres, 2001). 
Pág 187

El Modelo Integrativo de Turecki (2005) expone que el suicidio consumado está relacionado con dimensiones de impulsividad y agresividad, con factores biológicos que definen un endofenotipo de conducta suicida, con eventos traumáticos durante el periodo infantil y adulto, y con acumulación de experiencias negativas.
Otro factor relevante es el socioeconómico, pues como afirman Cervantes y Melo Hernández (2008), el desempleo y bajo nivel educativo son factores de riesgo para el suicidio, especialmente si la situación de escasez económica es prolongada (VegaPiñero, Blasco-Fontecilla, Baca-García, \& Díaz-Sastre, 2002), y dificulta el acceso a servicios de salud mental (Sánchez, Orejarena, \& Guzmán, 2004).

En estudios post mortem realizados a personas que se han suicidado, se ha encontrado factores neurobiológicos que pueden tener relación con el hecho, como un marcador de disminución de serotonina (metabolito $5 \mathrm{HIIA}$ ) en pacientes con depresión (Arango, Underwood, \& Mann, 2002; Sher et al., 2006), esquizofrenia y trastornos de personalidad (Gutiérrez \& Contreras, 2008), aumento de densidad de receptores (5-HT1A) (Mann, 2003), disminución de unión de trasportador de serotonina en la corteza prefrontal ventral (Arango et al., 2002; Kamally, Oquendo, \& Mann, 2001), el hipocampo (Pandeyet al., 2002) y la amígdala (Gutiérrez \& Contreras, 2008), cuyas estructuras participan en la experimentación de la emoción, manejo y afrontamiento del estrés, capacidad adaptativa ante eventos difíciles (Joiner et al., 2005), ansiedad y depresión (Blier \& Abbott, 2001).

El Modelo Integrativo de Turecki (2005) expone que el suicidio consumado está relacionado con dimensiones de impulsividad y agresividad, con factores biológicos que definen un endofenotipo de conducta suicida, con eventos traumáticos durante el periodo infantil y adulto, y con acumulación de experiencias negativas. Es importante resaltar que el daño cortical prefrontal genera desinhibición e impulsividad (Davidson, Putnam, \& Larson, 2000), asociadas a un alto grado de letalidad (Oquendo et al., 2003) y la alteración serotoninérgica también está altamente asociada a dificultades para regular ansiedad, impulsividad y agresión (Van Heeringen, 2003).

El modelo de diátesis-estrés hace referencia a una multicausalidad del riesgo suicida, el cual se da por la interacción de los genes y el ambiente (Gutiérrez-García, Contreras, \& Orozco-Rodríguez, 2006; Mann, Waternaux, Haas, \& Malone, 1999). Mann et al. (1999) sostienen la hipótesis de una trasmisión familiar, principalmente genética, de cierta propensión a externalizar la agresividad y una tendencia a presentar conductas suicidas. El riesgo suicida no está únicamente determinado por la posibilidad hereditaria de cierta enfermedad psiquiátrica sino también, y de manera primordial, por la tendencia a experimentar una mayor ideación suicida (Aranguren, 2009). La teoría de Mann (2003) se basa en el modelo médico de predisposición para un determinado trastorno y su aparición precipitada por factores como exacerbación de la enfermedad mental, una crisis vital y causas psicosociales.

Por otra parte, el modelo de trayectorias de desarrollo del suicidio de Silverman y Felner (1995) compara la conducta suicida con la historia evolutiva de un trastorno, suponiendo la existencia de una serie de procesos que conducen al suicidio y que se deben desplegar durante un determinado tiempo, y hacen uso del término vulnerabilidad personal, que resulta de la exposición a factores de riesgo y protectores que se pueden ir adquiriendo durante los diferentes ciclos etarios.

Respecto a factores psicológicos, la desesperanza se considera un importante predictor de suicidio en personas con trastornos mentales, y se asocia con consciencia de enfermedad (insight), creencias negativas sobre la enfermedad, percepción negativa del futuro y de sí mismo (Acosta et al., 2009; Brown et al., 2005; Henriques, Beck, \& Brown, 2003; López, F., López, F., \& López, S., 2008; Scholes \& Martin, 2013). Quintanilla Montoya, Haro Jiménez, Flores Villavicencio, Celis de la Rosa y Valencia Abundiz 
La presencia de un trastorno mental es un factor de riesgo de suicidio. Dentro de los diferentes trastornos, la depresión y la esquizofrenia aumentan significativamente el riesgo, siendo la desesperanza el componente más relacionado. Entre tanto, en las personas con trastornos de personalidad, ansiedad y consumo de sustancias, predominan la presencia de impulsividad y pérdida de control asociados al suicidio.
(2003), basados en la teoría de la indefensión aprendida de Seligman (1975), explican que la desesperanza ocurre cuando la persona interioriza impotencia o falta de control respecto a conductas o expectativas que son incongruentes con lo esperado. Al respecto, Sarmiento Falcón, Sánchez Sánchez, Vargas Polanco y Álvarez Rodríguez (2010) concluyen que "la desesperanza, comprendida como la sensación de imposibilidad absoluta de obtener una determinada cosa o de que ocurra algo que pueda cambiar la realidad existente, va generando impotencia y culpa que conduce al abandono de la lucha por la vida" (p. 5).

En un estudio realizado por Hawkins, Valencia, Caamaño y Ceballos (2014) con pacientes psiquiátricos hospitalizados, se encontró que el 60,7\% presentó riesgo de suicidio y el 70,4\% presentó desesperanza. El riesgo de ideación suicida y suicidio está determinado por la desesperanza junto con experiencias vitales negativas, falta de apoyo social, desmoralización, incapacidad para afrontar situaciones, aislamiento, conflicto y descuido (Caycedo et al., 2010; Joineret al., 2005), pérdida de control, sensación de desamparo y vacío, desesperación, ansiedad y pánico (Nizama, 2011). Beck, Brown, Berchick y Stewart (1990) enfatizan en la importancia de los aspectos cognitivos para comprender la conducta suicida, y proponen una hipótesis sobre el papel fundamental de la desesperanza como puente entre la depresión y el suicidio, por medio de una proyección cognitiva del estado actual depresivo en el futuro. De acuerdo con esta teoría, los acontecimientos negativos, sumados a un estilo atributivo interno provocarían déficit emocional, baja autoestima, aumento de los déficits cognitivos y de su cronicidad.

\section{Conclusiones}

La presencia de un trastorno mental es un factor de riesgo de suicidio. Dentro de los diferentes trastornos, la depresión y la esquizofrenia aumentan significativamente el riesgo, siendo la desesperanza el componente más relacionado. Entre tanto, en las personas con trastornos de personalidad, ansiedad y consumo de sustancias, predominan la presencia de impulsividad y pérdida de control asociados al suicidio. De otro lado, por sus implicaciones a nivel personal, familiar y social dicha problemática debe ser abordada con urgencia, y por su tendencia a aumentar tal como se ha registrado durante la última década en Colombia, esta situación se constituye en un problema de salud pública (Cendales, Vanegas, Fierro, Córdoba, \& Olarte, 2007); a lo que se suman las condiciones derivadas del conflicto armado, las cuales pueden agudizar factores como la desesperanza, los problemas familiares, económicos, el consumo de sustancias, etc., e incrementar el riesgo de suicidio.

En Colombia se comenzó a legislar en salud mental desde hace algunas décadas, pero es importante tener en cuenta que la prevención del suicidio y los trastornos mentales, además de la expedición de leyes y decretos, comprende actividades de educación, promoción de factores protectores, diagnóstico temprano, tratamiento eficaz e incluso control de condiciones medioambientales (Herrera, Ures, \& Martínez, 2015). Por tal motivo, es necesaria la intervención de profesionales debidamente capacitados en temas de salud general y salud mental, que permitan brindar una adecuada Atención Primaria de Salud (APS). También es preciso realizar seguimiento y control del tratamiento de los pacientes mediante una adecuada articulación entre los servicios de salud mental y los de salud general, para que la atención sea integral. Igualmente, es importante no sólo abordar los principales factores de salud/riesgo y sus interacciones, sino también los diferentes entornos vitales de cada población: individual, familiar y comunitario. 
Pág 189

Es fundamental "enseñar y desarrollar en grupos poblacionales específicos habilidades para identificar personas con alto riesgo suicida por medio de la educación sobre factores de riesgo, signos y señales de alarma, de manera que puedan ser referidas oportunamente para su tratamiento" (Arias López, 2013, p. 218), apoyados en un modelo de prevención primaria y secundaria en salud mental, con entrenamiento en afrontamiento y habilidades para resolver problemas, restricción de acceso a medios letales, tamizajes comunitarios y grupos de apoyo (Bobes, 2011).
Resulta primordial en la población con trastornos mentales y con conductas suicidas, estimular y fortalecer habilidades de comunicación, para resolver los problemas de manera adaptativa y buscar consejo y ayuda cuando surgen dificultades; la receptividad hacia las experiencias de otras personas, la autoconfianza, actitudes y valores positivos como el respeto, la solidaridad, cooperación, justicia y amistad (Jiménez et al., 2010); la autoestima, las creencias religiosas y las capacidades de autorregulación (Caycedo et al., 2010), el autocontrol y recuperación del equilibrio, así como el manejo del enojo y la tristeza (Rivera, 2010, p. 15). Del mismo modo, el fomento de estilos de crianza afectuosos contribuye a afianzar la autoestima, la seguridad y generan una participación social satisfactoria que previene la depresión y el comportamiento disocial (León \& Lainé, 2013).

Es fundamental "enseñar y desarrollar en grupos poblacionales específicos habilidades para identificar personas con alto riesgo suicida por medio de la educación sobre factores de riesgo, signos y señales de alarma, de manera que puedan ser referidas oportunamente para su tratamiento" (Arias López, 2013, p. 218), apoyados en un modelo de prevención primaria y secundaria en salud mental, con entrenamiento en afrontamiento y habilidades para resolver problemas, restricción de acceso a medios letales, tamizajes comunitarios y grupos de apoyo (Bobes, 2011). También es importante conocer las leyes de salud mental que permitan a la comunidad reconocer los derechos, deberes y procedimientos a seguir; en casos de ideación suicida tener una lista actualizada de contactos o instituciones donde se pueda acudir; en caso inminente de suicidio acordar con la persona en riesgo un plan por escrito y firmado de acción y preservación de la vida; e invitar a familiares y/o amigos a continuar en contacto no sólo durante el período crítico o posterior a éste sino también cuando la persona presente mejorías (World Federation for Mental Health, 2010).

En los casos en que se haya presentado un intento no letal, se recomienda un enlace inmediato con un profesional de salud mental que contemple el tratamiento con medicación y un proceso psicoterapéutico dirigido a tratar el intento de suicidio y el trastorno mental subyacente, si lo hay, con seguimiento constante y frecuente posterior al alta hospitalaria, dado que es un momento de gran vulnerabilidad. Igualmente, es recomendable que dicha alta no se efectúe hasta que el paciente se encuentre fuera de peligro y se trace un plan de salida integral en el que se implique a la familia, teniendo en cuenta su gran peso tanto como factor de riesgo como de protección.

\section{Referencias}

Abela, J., \& Seligman, M. (2000). The hopelessness theory of depression a test of the diathesis-stress component in the interpersonal and achievement domains. Cognit Ther Res, 24, 361-378. https://www.ncbi.nlm.nih.gov/pmc/articles/ PMC3883013/

Acosta, F., Aguilar, E., Cejas, M., Gracia, R., Caballero, A., \& Siris, S. (2009). A Prospective Study of the Psychopathological Variables Associated with Suicidality among Schizophrenic Patients. Actas Españolas de Psiquiatría, 37(1), 42-48. http://www. actasespanolasdepsiquiatria.es/repositorio/10/55/ENG/12816+10-1209.pdf

Addington, D.E. (2006). Seguridad en esquizofrenia: reducir el suicidio y el intento de suicidio. Revista de Toxicomanías, 49, 17-24. http://www.cat-barcelona.com/ uploads/rets/Ret49 3.pdf

Agosti, V., \& Levin, F. (2006). One-year follow up study of suicide attempters treated for drug dependence. Am J Addictions, 15, 293-296. http://www.tandfonline. $\mathrm{com} / \mathrm{doi} / \mathrm{abs} / 10.1080 / 10550490600754333$ ? needAccess=true 
Ajetunmobi, O., Taylor, M., Stockton, D., \& Wood, R. (2013). Early death in those previously hospitalised for mental healthcare in Scotland: A nationwide cohort study, 1986-2010. BMJ Open, doi: 10.1136/bmjopen-2013-002768.

Aliaga, J., Rodríguez, L., Ponce, C., Fristancho, A., \& Vereau, J. (2006). Escala de Desesperanza de Beck (bhs): Adaptación y características psicométricas. Revista de Investigación en Psicología, 9(1), 69-79. https://dialnet.unirioja.es/descarga/ articulo/2238209.pdf

American Psychiatric Association (APA). (1995). Manual Diagnóstico y Estadístico de los Trastornos Mentales DSM-IV. Barcelona: Masson. https://psicovalero.files. wordpress.com/2014/06/manual-diagnc3b3stico-y-estadc3adstico-de-los-trastornos-mentales-dsm-iv.pdf

American Psychiatric Association (APA). (2013). Diagnostic and Statistical Manual of Mental Disorders (5 Edition). Arlington, VA: American Psychiatric Association.

Andrade, J. (2012). Aspectos psicosociales del comportamiento suicida en adolescentes. Universidad Nacional Autónoma de México. Colombia. Recuperado de http:// www.scielosp.org/pdf/rsap/v13n1/v13n1a06

Arango, V., Underwood, M. D., \& Mann, J. J. (2002). Serotonin brain circuits involved in major depression and suicide. Prog Brain Res, 136, 443-453. http://psychiatryonline.org/pb/assets/raw/sitewide/practice guidelines/guidelines/bpd.pdf

Aranguren, M. (2009). Modelos teóricos de comprensión del suicidio.I Congreso Internacional de Investigación y Práctica Profesional en Psicología XVI Jornadas de Investigación. Quinto Encuentro de Investigadores en Psicología del Mercosur. Facultad de Psicología -Universidad de Buenos Aires, Buenos Aires.

Arbanas, G., Bicanic, S., Grba-Bujevic, M., \& Mlinac- Lucijanic, M. (2006). Suiciders, attempted suiciders and repeated attempted suiciders. Psychiatr Danub, 1, 128. http://revistas.ces.edu.co/index.php/psicologia/article/view/3503

Arias López, H. A. (2013). Factores de Éxito en Programas de Prevención del Suicidio. Revista Vanguardia Psicológica, 3(2), 215-225. https://dialnet.unirioja.es/descarga/articulo/4815157.pdf

Aristizábal, A., González, A., Palacio, C., García, J., \& López, C. (2009). Factores asociados a letalidad de intentos de suicidio en sujetos con trastorno depresivo mayor. Rev. Colomb Psiquiat, 38(3), 446 - 463. http://www.redalyc.org/ pdf/806/80615422005.pdf

Asociación Americana de Psiquiatría (APA). (2013). Guía de Consulta de los Criterios Diagnósticos del DSM V. Arlington, VA: Asociación Americana de Psiquiatría. http://www.saludypsicologia.com/posts/view/526/name:Guia-de-consulta-delos-criterios-diagnosticos-del-DSM-5

Baca, E., \& Aroca, F. (2014). Factores de riesgo de la conducta suicida asociados a trastornos depresivos y ansiedad. Salud Mental, 37, 373-380. http://www.redalyc.org/pdf/582/58232671003.pdf

Bakken, K., \& Vaglum, P. (2007). Predictors of suicide attempters in substance-dependent patients: a six-year prospective follow-up. Clin Pract Epidemiol Ment Health, 10, 3-20. https://www.ncbi.nlm.nih.gov/pmc/articles/PMC2098764/

Balhara, Y., \& Verma, R. (2012). Schizophrenia and Suicide. East Asian Archives of Psychiatry, 22(3), 126-133. https://www.ncbi.nlm.nih.gov/pubmed/23019287

Bartels, S. J., Coakley, E., Oxman, T. E., Constantino, G., Oslin, D., Chen, H.,... Sanchez, H. (2002). Suicidal and death ideation in older primary care patients with depression, anxiety, and at-risk alcohol use. American Journal of Geriatric Psychiatry, 10(4), 417-427. http://www.ajgponline.org/article/S1064-7481(12)61380-3/abstract 
Beck, A., Brown, G., Berchick, R., \& Stewart, B. (1990). Relationship between hopelessness and ultimate suicide: a replication with psychiatric outpatients. American Journal of Psychiatry, 147, 190-195. https://www.ncbi.nlm.nih.gov/ pubmed/2278535

Beghi, M., Rosenbaum, J. F., Cerri, C., \& Cornaggia, C. M. (2013). Risk factors for fatal and non fatal repetition of suicide attempts: A literature review. Neuropsychiatr Dis Treat, 9,1725-1736. https://www.ncbi.nlm.nih.gov/pubmed/24235836

Bernal, M., Haro, J. M., Bernet, S., Brugha, T., de Graaf, R., Bruffaerts, R., ... Alonso, J. (2007). Risk factors for suicidality in Europe: results from the ESEMED study. J Affect Disord, 101(1-3), 27-34. https://www.ncbi.nlm.nih.gov/pubmed/17074395

Bertolote, J. M, \& Fleischmann, A. (2002). Suicide and psychiatric diagnosis: a worldwide perspective. World Psychiatry, 1(3), 181-185. https://www.ncbi.nlm.nih. gov/pmc/articles/PMC1489848/

Bhatia, S. K., \& Bhatia, S. C. (2007). Childhood and adolescent depression. American Family Physician, 75(1), 74-80. Google books

Blier, P., \& Abbott, F. V. (2001). Putative mechanisms of action of antidepressant drugs in affective and anxiety disorders and pain. J Psychiatry Neurosci, 26(1), 37-43. https://www.ncbi.nlm.nih.gov/pubmed/11212592

Bobes, J. (2011). Suicidio y psiquiatría. Recomendaciones preventivas y de manejo del comportamiento suicida. Madrid: Editorial Triacastela. http://www.adamedfarma.es/wp-content/uploads/2015/05/Suicidio-y-psiquiatr\%C3\%ADa.-Recomendaciones-preventivas-y-de-manejo-del-comportamiento-suicida.pdf

Bolton, J. M., Belik, S. L., Enns, M. W., Cox, B. J., \& Sareen, J. (2008). Exploring the correlates of suicide attempts among individuals with major depressive disorder: findings from the national epidemiologic survey on alcohol and related conditions. J Clin Psychiatry, 69(7), 1139-1149. https://www.ncbi.nlm.nih.gov/ pubmed/18517287

Borges González, S.A., Vidal Palmer, L.E., \& Pérez Milan, J.F. (2006). Aspectos Medico legales de la Conducta Suicida. Rev. Hosp. Psiquiátrico de la Habana, 3(1), 1-7. Recuperado de http://www.revistahph.sld.cu/hph0106/hph011006.htm

Borges, G., Walters, E., \& Kessler, R. (2000). Associations of substance use, abuse, and dependence with subsequent suicidal behavior. Am J Epidemiol, 151, 781-789. http://aje.oxfordjournals.org/content/151/8/781

Borges, G., Wilcox, H. C., Medina-Mora, M. E., Zambrano, J., Blanco, J., \& Walters, E. (2005). Suicidal behavior in the Mexican National Comorbidity Survey (M-NCS): Lifetime and 12 month prevalence, psychiatric factors and service utilization. Salud Mental, 28(2), 40-47. http://new.medigraphic.com/cgi-bin/resumenl. cgi?IDARTICULO=6707

Brown, G. K., Ten, Th., Henriques, G. R., Xie, S. X., Hollander, J. E. \& Beck, A. T. (2005). Cognitive Therapy for the Prevention of Suicide Attempts. A Randomized Controlled Trial. Journal of the American Medical Association, 294, 563-570. https:// www.ncbi.nlm.nih.gov/pubmed/16077050

Cabra, O., Infante, D., \& Sossa, F. (2010). El suicidio y los factores de riesgo asociados en niños y adolescentes. Revista Médica Sanitas, 13(2), 28-35. http://www.unisanitas.edu.co/Revista/18/suicidio.pdf

Cantoral, D., \& Betancourt, D. (2011). Intento de suicidio y ambiente familiar en adolescentes de Tuxtla Gutiérrez, Chiapas. Revista de Educación y Desarrollo, 8(19),5966. http://www.funlam.edu.co/revistas/index.php/RCCS/article/view/1257

Carpenter, W., \& Buchanan, R. W. (1993). Domains of psychopathology relevant to the study of etiology and treatment in schizophrenia. En: Schulz, S.C., \&Taminga, C.A. (comps.). Schizophrenia: Cientific Progress. Oxford University Press, Nueva York. http://revistas.ces.edu.co/index.php/psicologia/article/view/3503 
Castro-Díaz, S., Gómez-Restrepo, C. Gil, F. Uribe Restrepo, M., Miranda, C., de la Espriella, M., Arenas, A., \& Pinto, D. (2013). Factores de riesgo para ideación suicida en pacientes con trastorno depresivo en Colombia. Rev Colomb Psiquiat, 43(1), 2735. http://www.elsevier.es/es-revista-revista-colombiana-psiquiatria-379-articulo-factores-riesgo-ideacion-suicida-pacientes-S0034745014000043

Cavanagh, J. T., Carson, A. J., Sharpe, M., Lawrie, S. M. (2003). Psychological autopsy studies of suicide: a systematic review. PsycholMed, 33(3), 395-405. https:// www.ncbi.nlm.nih.gov/pubmed/12701661

Caycedo, A., Arenas, M. L., Benítez, M., Cavanzo, P., Leal, G., \& Guzmán, Y. R. (2010). Características Psicosociales y Familiares Relacionadascon Intento de Suicidio en una PoblaciónAdolescente en Bogotá-2009. Persona y Bioética, 14(2), 205213. Recuperado de http://search.proquest.com/docview/865704238?account id $=44394$

Cendales, R., Vanegas, C., Fierro, M., Córdoba, R., \& Olarte, A. (2007). Tendencias del suicidio en Colombia, 1985-2002. Revista Panamericana de Salud Pública, 22(4), 231-238. http://www.scielosp.org/pdf/rpsp/v22n4/02.pdf

Centro Nacional de Memoria Histórica. (2013) ¡Basta Yaj Colombia: Memorias de guerra y dignidad. Recuperado de http://www.centrodememoriahistorica.gov.co/descargas/informes2013/bastaYa/resumen-ejecutivo-basta-ya.pdf

Cervantes, W., \& Melo Hernández, E. (2008). El suicidio en los adolescentes: Un problema en crecimiento. Duazary Revista de la Facultad de Ciencias de la Salud, Universidad del Magdalena, 5(2), 148-154. http://revistas.unimagdalena.edu.co/index.php/duazary/article/download/669/629

Chesney, E., Goodwin, G. M., \& Fazel, S. (2014). Risks of all-cause and suicide mortality in mental disorders: a meta-review. World Psychiatry, 13, 153-160. https:// www.ncbi.nlm.nih.gov/pubmed/24890068

Chioqueta, A., \& Stiles, T. (2003). Suicide risk in outpatients with specific mood and anxiety disorders. Crisis, 24(3), 105-112. http://econtent.hogrefe.com/doi/ abs/10.1027//0227-5910.24.3.105?journalCode=cri

Cifuentes, S.M. (2013). Comportamiento del suicidio, Colombia, 2013. Instituto Nacional de Medicina Legal y Ciencias Forenses. Forensis. Recuperado de https://www. medicinalegal.gov.co/documents/10180/188820/FORENSIS+2013+3-+suicidio. pdf/65a683b4-38b2-46a4-b32a-f2a0884b25bf

Cohen, B. (2006). Suicidio. En: Psiquiatría: teoría y práctica. Madrid-España. Editorial McGraw Hill-Interamericana. http://revistas.ces.edu.co/index.php/psicologia/ article/view/3503

Congreso de Colombia. (2013). Ley No 1616 de 2013 (21 Enero).Por medio de la cual se expide la ley de salud mental y se dictan otras disposiciones. Bogotá, D.C. Recuperado de http://wsp.presidencia.gov.co/Normativa/Leyes/Documents/2013/ LEY\%201616\%20DEL\%2021\%20DE\%20ENER0\%20DE\%202013.pdf

Conner, K., Beautrias, A., \& Conwell, Y. (2003). Risk factors for suicide and medically serious suicide attempts among alcoholics: analyses of Canterbury Suicide Project Data. J Stud Alcohol, 64, 551-555. https://www.ncbi.nlm.nih.gov/ pubmed/12921197

Davidson, R. J., Putnam, K. M., \& Larson, C. L. (2000). Dysfunction in the neural circuitry of emotion regulation -a possible prelude to violence. Science, 289, 591-594. https://www.ncbi.nlm.nih.gov/pubmed/10915615

Davis, L., Uezato, A., Newell, J. M., \& Frazier, E. (2008). Major depression and comorbid substance use disorders. Curr Opin Psychiatry, 21(1), 14-8. https://www.ncbi. nlm.nih.gov/pubmed/18281835 
De Hert, M., Correll, C. U., Bobes, J., Cetkovich-Bakmas, M., Cohen, D., Asai, I., ... Leucht, S. (2011). Physical illness in patients with severe mental disorders. I. Prevalence, impact of medications and disparities in health care. World Psychiatry, 10(1),52-

77. https://www.ncbi.nlm.nih.gov/pubmed/21379357

Departamento de Santander, Colombia. (2014). Proyecto de Ordenanza No 040 (31 de julio) Por medio de la cual se establecen los Lineamientos de la Política de Salud Mental y Convivencia Social del Departamento de Santander. Recuperado de http:// www.asambleadesantander.gov.co/index.php/component/jdownloads/send/4ordenanzas-2014/760-ordenanza-no-031-de-2014-1?option=com jdownloads

Dougherty, D. M., Mathias, C. W., Marsh, D. M., Moeller, F. G., \& Swann, A. C. (2004). Suicidal behaviors and drug abuse: impulsivity and its assessment. Drug Alcohol Depend, 76(Suppl), 93-105. https://www.ncbi.nlm.nih.gov/pubmed/15555820

Echávarri, O., Morales, S., Bedregal, P., Barros, J., Maino, M., Fischman, R., ... Moya, C. (2015). ¿Por Qué No Me Suicidaría? Comparación Entre Pacientes Hospitalizados en un Servicio de Psiquiatría con Distinta Conducta Suicida. PSYKHE, 24(1), 1-11.doi:10.7764/psykhe.24.1.667

Espinosa, J.J., Blum Grynberg, B., \& Romero Mendoza, M.P. (2009). Riesgo y letalidad suicida en pacientes con trastorno límite de la personalidad (TLP), en un hospital de psiquiatría. Salud Mental, 32(4), 317-325. http://new.medigraphic.com/ cgi-bin/resumenl.cgi?IDARTICULO $=21997$

Espinoza-Gómez, F. E., Zepeda-Pamplona, V. Z., Hernández-Suárez, C.M., NewtonSánchez, O. A., \& Plasencia-García, G. R. (2010). Violencia doméstica y riesgo de conducta suicida en universitarios adolescentes. Salud Publica Mex, 52(2), $213-$ 219. http://bvs.insp. $\mathrm{mx} / \mathrm{rsp} / \mathrm{articulos} / \mathrm{articulo.php?id=002463}$

Frías Ibañez, A., Vázquez Costa, M., del Real Peña, A., \& Sánchez del Castillo, C. (2012). Conducta autolesiva en adolescentes: prevalencia, factores de riesgo y tratamiento. Cuadernos de Medicina psicosomática y psiquiatría de enlace, 103, 33-48. http://repository.libertadores.edu.co/bitstream/11371/108/1/MoraSoledadMiryamRocio.pdf

Gairin, I., House, A., \& Owens, D. (2003). Attendance at the accident and emergency department in the year before suicide: retrospective study. Br J Psychiatr, 183, 28-33. https://www.ncbi.nlm.nih.gov/pubmed/12835240

Garlow, S. J., Purselle, D., \& D'Orio, B. (2003). Cocaine use disorders and suicidal ideation. Drug Alcohol Depend, 70(1), 101-104. https://www.ncbi.nlm.nih.gov/pubmed/12681530

Goldacre, M., Seagroatt, V., \& Hawton, K. (1993). Suicide after discharge from psychiatric inpatient care. Lancet, 342(8866), 283 - 286. http://www.thelancet.com/ journals/lancet/article/PII0140-6736(93)91822-4/abstract

González, J. E., \& Camejo, Z.V. (2014). Ideación suicida, diagnóstico psiquiátrico y factores de riesgo suicida en pacientes adolescentes con conducta suicida. Alcmeon, Revista Argentina de Clínica Neuropsiquiátrica, 19(1), 62 - 78. http://www. alcmeon.com.ar/19/09 suicidioadolesc gonzalez.pdf

González-Quiñones, J., \& De la Hoz Restrepo, F. (2010). Relaciones entre los comportamientos de riesgo psicosociales y la familia en adolescentes de Suba, Bogotá. Revista de Salud Pública, 13(1), 67-78. Recuperado de http://www.scielosp.org/pdf/ $\underline{\mathrm{rsap} / \mathrm{v} 13 \mathrm{n} 1 / \mathrm{v} 13 \mathrm{n} 1 \mathrm{a} 06}$

Goodwin, F. K., \& Jaminson, K. R. (2007). Manic-Depressive Illness: Bipolar disorders and recurrent depression, 2nd edn. New York: Oxford University Press. https://global.oup.com/academic/product/manic-depressive-illness9780195135794?cc=us\&lang=en\&

Guibert Reyes, W., \& Niurka Torres, M. (2001). Intento suicida y funcionamiento familiar. Rev Cubana Med Gen Integr, 17(5), 452 - 460. http://www.bvs.sld.cu/revistas/ mgi/vol17 5 01/mgi08501.pdf 
Guo, B., \& Harstall, C. (2002). Efficacy of suicide prevention programs for children and youth. Edmonton, AB, Canada: Alberta Heritage Foundation for Medical Research. Health Technology Assessment; 26 Series A. https://www.ncbi.nlm.nih. gov/books/NBK69303/

Gutiérrez, A.G., \& Contreras, C.M. (2008). El suicidio y algunos de sus correlatos neurobiológicos. Segunda parte. Salud Mental, 31, 417-425. http://www.redalyc.org/ pdf/582/58231509.pdf

Gutiérrez-García, A. G., Contreras, C. M., \& Orozco-Rodríguez, R. C. (2006). El suicidio: conceptos actuales. Salud Mental, 29(5), 66-74. http://www.redalyc.org/ pdf/582/58229510.pdf

Hagnell, O., Lanke, J., Rorsman, B., \& Ojesjo, L. (1982) ¿Estamos entrando en una era de lamelancolía? La enfermedad depresiva en un estudio epidemiológico prospectivo de más de 25 años: El estudio Lundby. Medicina Psicológica, 12, 279-289.

Hansagi, H., Olsson, M., Hussain, A., \& Onlén, G. (2008). Is information sharing between the emergency department and primary care useful to the care of frequent emergency department users? Eur J Emerg Med, 15(1), 34- 39. https:// www.ncbi.nlm.nih.gov/pubmed/18180664

Harkavy-Friedman, J. M., Restifo, K., Malaspina, D., Kaufmann, C. A., Amador, X. F., Tale, S. A., \& Gorman, J. M. (1999). Suicidal behavior in schizophrenia characteristics of individuals who had and had not attempted suicide. Am J Psychiat, 156(8), 1276-1278. https://www.ncbi.nlm.nih.gov/pubmed/10450275

Harwitz, D., \& Ravizza, L. (2000). Suicide and depression. Emerg Med Clin North Am, 18(2), 263-271, ix.

Hausmann, A., \& Wolfgang, F. (2000). Depression in patients with schizophrenia. CNS Drugs, 14(4), 289 - 299. http://link.springer.com/article/10.2165/00023210-200014040-00004

Hawkins, S., Valencia, A., Caamaño, B., \& Ceballos, C. (2014). Riesgo suicida y desesperanza en pacientes psiquiátricos hospitalizados. Pensando Psicología, 10(17), 43-51. doi: http://dx.doi.org/10.16925/pe.v10i17.783

Hawton, K., \& Van Heeringen, K. (2009). Suicide. Lancet, 373(9672), 1372-1381. http:// www.thelancet.com/journals/lancet/article/PIIS0140-6736(09)60372-X/abstract

He, X. Y., Felthous, A. R., Holzer, C. E., Nathan, P., \& Veasey, S. (2001). Factors in prison suicide: one year study in Texas. J Forensic Sci, 46(4), 896-901. https://www. ncbi.nlm.nih.gov/pubmed/11451074

Healy, D., \& Alfred, G. (2005). Antidepressant drug use \& the risk of suicide. Int Rev Psychiatry, 17(3),163-172. https://www.ncbi.nlm.nih.gov/pubmed/16194787

Henriques, G., Beck A.T., \& Brown, G. (2003). Cognitive Therapy for Adolescent and Young Adult Suicide Attempters. The American Behavioral Scientist, 46(9),12581268. https://www.researchgate.net/publication/238335072 Cognitive Therapy for Adolescent and Young Adult Suicide Attempters

Hernández, A., González-Elías, I. E., \& López, Y. M. (2013). Factores de riesgo relacionados con la conducta suicida en la infancia y adolescencia. MEDISAN, 17(12), 9027- 9035. http://www.bvs.sld.cu/revistas/san/vol17_12_13/san011712.pdf

Herrera, R., Ures, M., \& Martínez, J. (2015). El tratamiento del suicidio en la prensa española: ¿Efecto Werther o efecto papageno? Revista Asociación Española Neuropsiquica, 35(125), 123-134. http://www.redalyc.org/pdf/2650/265032967009. $\underline{\mathrm{pdf}}$

Ho, T.P. (2003). The suicide risk of discharged psychiatric patients. J Clin Psychiatry, 64. 702-707. http://www.psychiatrist.com/JCP/article/Pages/2003/v64n06/ v64n0613.aspx 
Hoang, U., Stewart, R., \& Goldacre, M. J. (2011). Mortality after hospital discharge for people with schizophrenia or bipolar disorder: retrospective study of linked English hospital episode statistics, 1999-2006. BMJ, 343, d5422. http://www.bmj. com/content/343/bmj.d5422

Hor, K., \& Taylor, M. (2010). Review: suicide and schizophrenia: a systematic review of rates and risk factors. J Psychopharmacol, 24(4), 81-90. https://www.ncbi.nlm. nih.gov/pmc/articles/PMC2951591/

Hunt, I., Kapur, N., Robinson, J., Shaw, J., Flynn, S., Bailey, H., ... Appleby, L. (2006a). Suicide within 12 months of mental health service contact in different age and diagnostic groups. Br J Psychiatry, 188, 135-142. http://bjp.rcpsych.org/content/188/2/135

Hunt, I., Kapur, N., Windfuhr, K., Robinson, J., Bickley, H., Flynn, S., ...Appleby, L. (2006b). Suicide in schizophrenia: findings from a national clinical survey. J Psychiatr Pract, 12(3), 139-147. https://www.ncbi.nlm.nih.gov/pubmed/16732132

Hunt, I., Kapur, N., Webb, R., Robinson, J., Burns, J., Shaw, J., ... Windfuhr, K. (2009). Suicide in recently discharged psychiatric patients: a case control study. Psychol Med, 39(3), 443-439. https://www.ncbi.nlm.nih.gov/pubmed/18507877

Jiménez, J., Martín, S., Pacheco, T., Pérez-Iñigo, J., Robles, J., Santiago, P., \& Torras, A. (2010). Guía para Familiares. Detección y prevención de la conducta suicida en personas con una enfermedad mental. Madrid, España: Comunidad de MadridFEMASAM. http://www.madrid.org/bvirtual/BVCM017097.pdf

Joiner, T. E., Brown, J. S., \& Wingate, L. R. (2005). The psychology and neurobiology of suicidal behavior. Annu Rev Psychol, 56, 287-314. http://www.annualreviews. org/doi/abs/10.1146/annurev.psych.56.091103.070320

Kamally, M., Oquendo, M.A., \& Mann, J.J. (2001). Understanding the neurobiology of suicidal behavior. Depress Anxiety, 14(3), 164-176. https://www.ncbi.nlm.nih.gov/pubmed/11747126

Kessler, R.C., Berglund, P., Borges, G., Nock, M., \& Wang, P.S. (2005). Trends in suicide ideation, plans, gestures, and attempts in the United States, 1990-1992 to 2001 2003. JAMA, 293, 2487-2495. https://www.ncbi.nlm.nih.gov/pubmed/15914749

Labonté, B., Suderman, M., Maussion, G., Lopez, J., Navarro- Sánchez, L., Yerko, V., ... Turecki, G. (2013). Genome-Wide Methylation Changes in the Brains of Suicide Completers. American Journal Psychiatry, 170(5), 511-520. https://www.ncbi. nlm.nih.gov/pubmed/23511308

Labonté, B., Yerko, V., Gross, J., Mechawar, N., Meaney, M., Szyf, M., \& Turecki, G. (2012). Differential Glucocorticoid Receptor Exon 1(B), 1(C), and 1(H) Expression and Methylation in Suicide Completers with a History of Childhood Abuse. Biological Psychiatry, 72(1), 41-48. http://www.biologicalpsychiatryjournal.com/article/ S0006-3223(12)00132-1/abstract

Lecrubier, Y. (2002). La influencia de la comorbilidad en la prevalencia de la conducta suicida. Eur Psychiatry Ed. Esp, 9, 90-94. http://formaciones.elmedicointeractivo. com/emiold/publicaciones/europeanpsy2002/2/90-94.pdf

LeGris, J., \& van Reekum, R. (2006). The neuropsychological correlates of borderline personality disorder and suicidal behavior. Can J Psychiatry, 51(3), 131-142. https://ww1. cpa-apc.org/Publications/Archives/CJP/2006/march1/cjp-mar-1-06-legris-IR.pdf

Lejoyeux, M., \& Marinescu, M. (2006). Alcohol dependence and abuse and psychiatric disorders. Rev Prat, 56(10), 1081-1085. https://www.ncbi.nlm.nih.gov/ pubmed $/ 16836203$

León, S., \& Lainé, D. (2013). Conducta suicida en adolescentes. Congreso Internacional de la niñez, adolescencia y familia. III CongresoCubano y Caribeño de atención integral a la salud de la adolescencia. VI Seminario Iberoamericano de adolescencia y juventud (CODAJIC). Cuba: Hospital Pediátrico Docente Juan M. Márquez. http://www.codajic.org/ sites/www.codajic.org/files/20\%20-\%20CONDUCTA\%20SUICIDA\%20\%20EN\%20 ADOLESCENTES..pdf 
López, F., López, F., \& López, S. (2008). Ideación suicida y desesperanza en pacientes psiquiátricos hospitalizados. Alcmeon, Revista Argentina de Clínica Neuropsiquiátrica, 14(3), 33-41. http://www.alcmeon.com.ar/14/55/06 lopez.pdf

Luoma, J. B., Martin, C. E., \& Pearson, J. L. (2002). Contact with Mental Health and Primary Care Providers before Suicide: A Review of the Evidence. Am J Psychiatry, 159, 909-916. https://www.ncbi.nlm.nih.gov/pubmed/12042175

Mann, J. (2003). Neurobiology of suicidal behaviour. Nat Rev Neurosci, 4(10), 819-828. https://www.ncbi.nlm.nih.gov/labs/articles/14523381/

Mann, J. J., Waternaux, C., Haas, G.L., \& Malone, K. M. (1999). Toward a Clinical Model of Suicidal Behavior in Psychiatric Patients. American Journal of Psychiatry, 156, 181-189. https://www.ncbi.nlm.nih.gov/pubmed/9989552

Mardomingo, M.J. (2000). Trastornos de personalidad y comportamiento suicida en la adolescencia.I Congreso Virtual de Psiquiatría 1 de Febrero - 15 de Marzo 2000 [citado: 21/07/2015]; Conferencia 7-Cl-H: [8 pantallas]. Disponible en:http:// www.psiquiatria.com/congreso/mesas/mesa7/conferencias/7-ci-h.htm

Maris, R.W. (2002). Suicide. Lancet, 360(9329), 319-26. https://www.ncbi.nlm.nih. gov/pubmed/12147388

Matschnig, T., Fruhwald, S., \& Frottier, P. (2006). Suicide behind bars -an international review. Psychiatr Prax, 33(1), 6-13.

Mejía, M., Sanhueza, P., \& González, J. (2011). Factores de riesgo y contexto del suicidio. Revista Memoriza.com, 8, 15-25. http://www.memoriza.com/documentos/ revista/2011/Suicidi02011 8 15-25.pdf

Meltzer, H.Y. (1998). Suicide in schizophrenia risk factors and clozapine treatment. J ClinPsychiatry, 59(3), 15-20. https://www.ncbi.nlm.nih.gov/pubmed/9541333

Mendieta, D.C. (1997). La conducta autolesiva en el trastorno límite de la personalidad. Instituto Nacional Psiquiatría. Inf Clin, 8(5), 28-29. http://www.scielo.org.

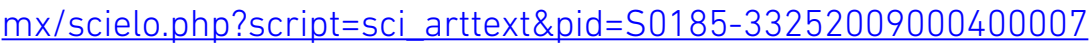

Miles, C. (1997). Condition predisposing to suicide: a review. J Nerv Ment Dis, 164 231-246. https://www.ncbi.nlm.nih.gov/pubmed/321725

Ministerio de Salud República de Colombia. (1998). Resolución Nª 2358 de 1998 (Junio 18). Política Nacional de Salud Mental. Santa Fe de Bogotá, D.C. Recuperado de https://www.minsalud.gov.co/Normatividad Nuevo/RESOLUCI\%C3\%93N\%20 2358\%20DE\%201998.pdf

Ministerio de la Protección Social República de Colombia \& Fundación FES Social. (2005). Lineamientos de Política de Salud Mental para Colombia. Cali, Colombia. Recuperado de https://www.minsalud.gov.co/Lineamientos/Lineamientos\%20 -Pol\%C3\%ADtica\%20Salud\%20Mental.pdf

Ministerio de Salud y Protección Social República de Colombia. (2011). Boletín de Prensa No 316 de 2011. Ministerio presentó Observatorio Nacional de Salud Mental. Recuperado de https://www.minsalud.gov.co/Paginas/Ministeriopresent\%C3\%B3-Observatorio-Nacional-de-Salud-Mental-.aspx

Ministerio de Salud y Protección Social República de Colombia \& Organización Panamericana de la Salud (OPS/OMS). (2014). Documento Propuesta de Ajuste de la Política Nacional de Salud Mental para Colombia 2014. Plan Nacional de Salud Mental 2014 - 2021. Recuperado de http://www.consejonacionaldetrabajosocial.org.co/cnts/images/POLITICA-NACIONAL-DE-SALUD-MENTAL-2014.pdf

Ministerio de Salud y Protección Social República de Colombia. (2014). Programa Nacional de Salud y Medicina Familiar y Comunitaria. Dirección de Desarrollo del Talento Humano en Salud. Recuperado de https://www.minsalud.gov.co/sites/ rid/Lists/BibliotecaDigital/RIDE/VS/TH/Programa\%20Nacional\%20Salud\%20 Familiar\%20y\%20Comunitaria\%20y\%20Medicina\%20Familiar\%20200214.pdf 
Ministerio de Salud y Protección Social, COLCIENCIAS, Pontificia Universidad Javeriana, Datos, Procesos y Tecnología SAS. (2015). Encuesta Nacional de Salud Mental. Tomo 1. Recuperado de http://www.odc.gov.co/Portals/1/publicaciones/pdf/ consumo/estudios/nacionales/C0031102015-salud mental tomol.pdf

Ministerio de Salud y Protección Social. (2016). Política de Atención Integral en Salud. Bogotá D.C. Recuperado de https://www.minsalud.gov.co/sites/rid/Lists/BibliotecaDigital/RIDE/DE/20160216-presentacion-mias.pdf

Muñoz, J., Pinto, V., Callata, H., Napa, N., \& Perales, A. (2005). Ideación suicida y cohesión familiar en estudiantes preuniversitarios entre 15 y 24 años, Lima 2005. Revista Perú Medicina Experimental de Salud Pública, 23(4) 239-246. http://www. scielo.org.pe/scielo.php?pid=S1726-46342006000400002\&script=sci abstract

Nemeroff, C. B., Compton, M. T., \& Berger, J. (2001). The depressed suicidal patient. Assessment and treatment. Ann N Y Acad Sci, 932, 1-23. https://www.ncbi.nlm. nih.gov/pubmed/11411180

Nizama, M. (2011). Suicidio. Rev Peru Epidemiol, 15(2), 5 pp. https://dialnet.unirioja. es/descarga/articulo/3994798.pdf

Nock, M. K., Borges, G., Bromet, E. J., Cha, C. B., Kessler, R.C., \& Lee, S. (2008). Suicide and suicidal behavior. Epidemiol Rev, 30, 133-154. https://www.ncbi.nlm. nih.gov/pubmed/18653727

Norton, P. J., Temple, S. R., \& Pettit, J. W. (2008). Suicidal ideation and anxiety disorders: elevated risk or artifact of comorbid depression? J Behav Ther Exp Psychiatry, 39(4),515-25. https://www.ncbi.nlm.nih.gov/pubmed/18294614

Ocampo, R., Bojorquez, I., \& Cortés, M. (2009). Consumo de sustancias y suicidios en México: resultados del Sistema de Vigilancia Epidemiológica de las Adicciones, 1994-2006. Salud Publica Mex, 51, 306-313. http://www.scielo.org.mx/pdf/spm/ v51n4/v51n4a07.pdf

Olié, E., Guillaume, S., Jaussent, I., Courtet, P., \& Jollant, F. (2010). Higher psychological pain during a major depressive episode may be a factor of vulnerability to suicidal ideation and act. J Affect Disord, 120, 226-230. https://www.ncbi.nlm. nih.gov/pubmed/19394086

Oquendo, M. A., Placidi, G. P., Malone, K. M., Campbell, C., Keilp, J., Brodsky, B., ... Mann, J. J. (2003). Positron emission tomography of regional brain metabolic responses to a serotonergic challenge and lethality of suicide attempts in major depression. Arch Gen Psychiatry, 60(1), 14-22. https://www.ncbi.nlm.nih.gov/ pubmed/12511168

Organización Mundial de la Salud (OMS). (2012a). Guía de intervención mhGAP para los trastornos mentales, neurológicos y por uso de sustancias en el nivel de atención de la salud no especializada ( $2^{a}$ reimpresión al español corregida). Ginebra: OMS. Recuperado de http://apps.who.int/iris/bitstream/10665/44498/1/9789243548067 spa.pdf

Organización Mundial de la Salud (OMS). (2012b). Salud mental: prevención del suicidio (SUPRE).Ginebra, Suiza: OMS. Recuperado de http://www.who.int/es/

Organización Mundial de la Salud (OMS). (2013). Plan de acción sobre salud mental 2013-2020. Ginebra, Suiza: OMS. Recuperado de http://apps.who.int/iris/bitstre am/10665/97488/1/9789243506029 spa.pdf

Organización Panamericana de la Salud (OPS). (2003). Atención Primaria en Salud en las Américas: Enseñanzas extraídas a lo largo de 25 años y retos futuros. Washington, D.C.: OPS. http://iris.paho.org/xmlui/handle/123456789/7479

Palacio, C., García, J., Diago, J., Zapata, C., López, G., Ortiz,J.,\& Lopez, M. (2007). Identification of suicide risk factors in Medellin, Colombia: a case-control study of psychological autopsy in a developing country. Arch Suicide Res, 11(3), 297-308. https://www.ncbi.nlm.nih.gov/pubmed/17558615 
Pan, A., Keum, N., Okereke, O. I., Sun, Q., Kivimaki, M., Rubin, R. R., \& Hu, F. B. (2012). Bidirectional association between depression and metabolic syndrome: A systematic review and meta-analysis of epidemiological studies. Diabetes Care, 35(5), 1171-1180. http://care.diabetesjournals.org/content/35/5/1171.abstract

Pandey, G. N., Dwivedi, Y., Rizavi, H. S., Ren, X., Pandey, S. C., Pesold, C., ... Tamminga, C. A. (2002). Higher expression of serotonin 5-HT (2A) receptors in the postmortem brains of teenage suicide victims. Am J Psychiatry, 159, 419-429. https://www. ncbi.nlm.nih.gov/pubmed/11870006

Park, H. S., Schepp, K. G., Jang, E. H., \& Koo, H. Y. (2006). Predictors of suicidal ideation among high school students by gender in South Korea. J Sch Health, 76, 181-188. https://www.ncbi.nlm.nih.gov/pubmed/16635202

Pavez, P., Santander, N., Carranza, J., \& Vera-Villaroel, P. (2009). Factores de riesgo familiares asociados a la conducta suicida en adolescentes con trastorno depresivo. Revista Médica de Chile, 137(2), 226-233. http://www.scielo.cl/scielo. php?script=sci arttext\&pid=S0034-98872009000200006

Pelkonen, M., \& Marttunen, M. (2003). Child and adolescent suicide. Epidemiology, risk factors, and approaches to prevention. Pediatr Drugs, 5(4), 243-265. http:// link.springer.com/article/10.2165/00128072-200305040-00004

Pérez, J. (2011). La Mirada del Suicida: El Enigma y el Estigma. Plaza y Valdés Editores. Madrid. http://www.plazayvaldes.es/libro/la-mirada-del-suicida

Phillips, M.R. (2010). Rethinking the role of mental illness in suicide. Am J Psychiatry, 167, 731-733. https://www.researchgate.net/publication/44901917 Rethinking the Role of Mental Illness in Suicide

Post, R. (2005). The impact of bipolar depression. Journal of Clinical Psychiatry, 66(5), 5-10. https://www.ncbi.nlm.nih.gov/pubmed/16038596

Preuss, U., Schuckit, M., Smith, T., Danko, G., Bucholz, K., Hesselbrock, M., ... Kramer, J.R.(2002). Predictors and correlates of suicide attempts over 5 years in 1,237 alcohol-dependent men and women. Am J Psychiatry, 160, 53-56. https://www. ncbi.nlm.nih.gov/pmc/articles/PMC2098764/

Qin, P. (2011). The impact of psychiatric illness on suicide: Differences by diagnosis of disorders and by sex and age of subjects. J Psychiatr Res, 45(11), 1445-1152. https://www.ncbi.nlm.nih.gov/pubmed/21722920

Quintanilla Montoya, R., Haro Jiménez, L. P., Flores Villavicencio, M. E., Celis de la Rosa, A., \& Valencia Abundiz, S. (2003). Desesperanza y tentativa suicida. Investigación en Salud, 5(2), 113-116. http://www.scielo.org.pe/scielo.php?pid=S0254$92472011000200005 \&$ script $=$ sci arttext

Ramírez, L.A., \& Naranjo, C.A. (2014). Comportamiento del suicidio. Colombia, 2014. En Instituto Nacional de Medicina Legal y Ciencias Forenses. Forensis. Datos para la vida. Herramienta para la interpretación, intervención y prevención de lesiones a causa externa en Colombia, 16(1), 319 - 351. http://www.medicinalegal.gov.co/.../ Forensis...2014.../9085ad79-d2a9-4c0d-a17b-f845ab9...

Rascón, M. L., Gutiérrez, M., Valencia, M., Díaz, L. R., Leaños, C., \& Rodríguez, S. (2004). Percepción de los familiares del intento e ideación suicidas de pacientes con esquizofrenia. Salud Mental, 27(5), 44-52. http://new.medigraphic.com/cgi-bin/resumen.cgi?IDARTICULO=16597

Rey Sarmiento, F. (2009). Modelo de Gestión Operativa para el Componente de Salud Mental en Atención Primaria en Salud. Bogotá, D.C: Ministerio de la Protección Social República de Colombia. https://www.minsalud.gov.co/rea\%20de\%20trabajo\%20 colaborativo/Atenci\%C3\%B3n\%20primaria\%20en\%20salud\%20-\%20APS/DOCUMENTO\%2OFINAL\%20MODEL0\%20GESTI\%C3\%93N.pdf

Rivera, M. (2010). Psicología y Comunicación Visual. Estrategias para la prevención del suicidio en adolescentes. México: Trillas. https://www.ceapa.es/sites/default/files/ uploads/ficheros/publicacion/prevencion del suicidio en adolescentes si.pdf 
Rodríguez, M., \& Guerrero, S. (2005). Frecuencia y fenomenología de lesiones autoinfligidas en mujeres colombianas con trastornos del comportamiento alimentario. Revista Colombiana de Psiquiatría, 34(3), 333-354. http://www.redalyc.org/ pdf/806/80634302.pdf

Rodríguez, A. (2007). El suicidio y su prevención. En Instituto Nacional de Medicina Legal Medicina Legal y Ciencias Forenses - INML y CF (2006). 275-282. Recuperado de: http://www.medicinalegal.gov.co/documents/10180/33850/8+Suici dios.pdf/088bf4a4-b9d5-4246-a4e7-3298e9c0f05d

Saha, S., Chant, D., \& McGrath, J. (2007). A systematic review of mortality in schizophrenia: is the differential mortality gap worsening overtime? Arch Gen Psychiatry, 64, 1123-1131. https://www.ncbi.nlm.nih.gov/pubmed/17909124

Salirrosas-Alegría, C., \& Saavedra-Castillo, J. (2014). Percepción de algunos estilo de crianza y el episodio depresivo en el adulto. Rev Neuropsiquiatra, 77(3), 160-167. http://www.redalyc.org/pdf/3720/372033987004.pdf

Samuels, J. F., Nestadt, G., Romanoski, A. J., Folstein, M. F., \& McHugh, P. R. (1994). DSM-III personality disorders in the community. Am J Psychiatry, 151, 1055-1062. https://www.ncbi.nlm.nih.gov/pubmed/8010364

Sánchez, R., Orejarena, S., \& Guzmán, Y. (2004). Características de los Suicidas en Bogotá: 1985-2000. Rev Salud Pública, 6(3), 217-234. http://www.scielosp.org/ $\mathrm{pdf} / \mathrm{rsap} / \mathrm{v} 6 \mathrm{n} 3 / \mathrm{a} 01 \mathrm{v} 6 \mathrm{n} 3 . \mathrm{pdf}$

Santamarina, S., Iglesias, C., \& Alonso, M. (2004). Comportamientos suicidas y consumo de sustancias psicótropas. En J., Bobes,P., Sáiz, M., García-Portilla, M., Bascarán, \& M. Bousoño, Comportamientos suicidas, prevención y tratamiento. Barcelona: Ars Médica, 91-95.

Sareen, J., Cox, B. J., Afifi, T. O., de Graaf, R., Asmundson, G. J., ten Have, M., \& Stein, M. B. (2005). Anxiety disorders and risk for suicidal ideation and suicide attempts: a population-based longitudinal study of adults. Arch Gen Psychiatry, 62(11), 12491257. https://www.ncbi.nlm.nih.gov/pubmed/16275812

Sarmiento Falcón, Z., Sánchez Sánchez, S., Vargas Polanco, I., \& Álvarez Rodríguez, M. (2010). Conducta suicida y su relación con los factores de riesgo psicosociales. Recuperado de bvs.sld.cu/revistas/san/vol 148 10/san02810.htm

Scholes, B., \& Martin, C. (2013). Measuring depression in schizophrenia with questionnaires. Journal of Psychiatric and Mental Health Nursing, 20(1), 17-22. doi: 10.1111/j.1365-2850.2012.01877.x

Schwartz, R. (2000). Insight and suicidality in schizophrenia: A replication study. $J$ Nerv Ment Dis, 188, 235 - 236. http://www.redalyc.org/pdf/582/58252706.pdf

Seligman, M. E. P. (1975). Helplessness: On Depression, Development, and Death. San Francisco: W. H. Freeman. https://www.amazon.es/Helplessness-DepressionDevelopment-Death-Psychology/dp/071672328X

Sharma, V. (2003). Atypical antipsychotics and suicide in mood and anxiety disorders. Bipolar Disorder, 5(2), 48-52. https://www.ncbi.nlm.nih.gov/pubmed/14700012

Sher, L., Mann, J. J., Traskman-Bendz, L., Winchel, R., Huang, Y. Y., Fertuck, E., \& Stanley, B. H. (2006). Lower cerebrospinal fluid homovanillic acid levels in depressed suicide attempters.J Affect Disord, 90(1), 83-89. https://www.researchgate.net/ publication/7459209 Lower cerebrospinal fluid homovanillic acid levels in depressed suicide attempters

Silva, D., Vicente, B., Saldivia, S., \& Kohn, R. (2013). Conducta suicida y trastornos psiquiátricos en Chile, un estudio poblacional. Rev Med Chile, 141, 1275-1282. http:// www.scielo.cl/scielo.php?script=sci arttext\&pid=S0034-98872013001000006

Silverman, M., \& Felner, R. (1995). The place of suicide prevention in the spectrum of intervention: definitions of critical terms and constructs. Suicide Life Threat Behav. World Psychiatry, 3(3), 152-153. https://www.ncbi.nlm.nih.gov/pmc/articles/PMC1414696/ 
Sodeberg, S. (2001). Personality disorders in parasuicide. Nordic J Psychiatry, 55, 163-167. https://www.ncbi.nlm.nih.gov/pubmed/11827610

Soloff, P. H., Fabio, A., Kelly, T. M., Malone, K. M., \& Mann, J.J. (2005). High-lethality status in patients with borderline personality disorder. J Pers Disord, 19(4), 386399. https://www.ncbi.nlm.nih.gov/pubmed/16178681

Soloff, P., Lynch, K., \& Kelly, T. (2002). Childhood abuse as a risk factor suicidal behavior borderline personality disorder. J Personal Disord, 16, 201-14. Google books

Steele, M. M., \& Doey, T. (2007). Suicidal behaviour in children and adolescents. Part 1: etiology and risk factors. Can J Psychiatry, 52(1), 21S-33S. https://www.ncbi. nlm.nih.gov/pubmed/17824350

Strakowski, S. M., McElroy, S. L., Keck, P. E., \& West, S. A. (1996). Suicidality among patients with mixed and manic bipolar disorder. Am J Psychiatry, 153, 674-676. Google books

Swann, A. E., Lijffijt, M., Lane, S. D., Steinberg, J. L., \& Moeller, F. G. (2009). Increasedtrait-like impulsivity and course of illness in bipolar disorder. Bipolar Disorder, 11(3), 280-288. https://www.ncbi.nlm.nih.gov/pmc/articles/PMC2723745/

Tondo, L., Hennen, J., \& Baldessarini, R. J. (2001). Lower suicide risk with long-term lithium treatment in major affective illness: a meta-analysis. Acta Psychiatr Scand, 104(3), 163-172. https://www.ncbi.nlm.nih.gov/pubmed/11531653

Toro, D., Paniagua, R., González, C., \& Montoya, B. (2009) Caracterización de adolescentes escolarizados con riesgo de suicidio, Medellín, 2006. Rev. Fac. Nac. Salud Pública, 27(3), 302-308. http://www.scielo.org.co/scielo.php?script=sci arttext\&pid...386X2009000300007

Turecki, G. (2005) Dissecting the suicide phenotype: the role of impulsive-aggressive behaviours. J Psychiatry Neurosci, 30(6), 398-408. https://www.ncbi.nlm.nih. gov/pmc/articles/PMC1277022/

Valadez-Figueroa, I., Amezcua-Fernández, R., Quintanilla-Montoya, R., \& GonzálezGallegos, N. (2005). Familia e intento suicida en el adolescente de educación media superior. Archivos en Medicina Familiar, 7(3), 69-78. http://www.redalyc. org/pdf/507/50730702.pdf

Van Heeringen, K. (2003). The neurobiology of suicide and suicidality. Can J Psychiatry, 48(5), 292-300. https://www.ncbi.nlm.nih.gov/pubmed/12866334

Vega-Piñero, M., Blasco-Fontecilla, H., Baca-García, E., \& Díaz-Sastre. C. (2002). El suicidio. Salud Global, 2(4), 2-16. http://www.who.int/mental health/prevention/suicide/suicideprevent/es/

Verona, E., Patrick, C. J., \& Joiner, T. W. (2001). Psychopathy, antisocial personality, and suicide risk. J Abnorm Psychol, 110(3), 462-470. https://www.ncbi.nlm.nih. gov/pubmed/11502089

Williams, E.B. (1992). The psychological treatment of depression: A guide to the theory and practice of cognitive behavior therapy. Routledge, Londres. http://onlinelibrary.wiley.com/doi/10.1002/casp.2450050108/abstract

Windfuhr, K., \& Kapur, N. (2011). Suicide and mental illness: a clinical review of 15 years findings from the UK National Confidential Inquiry into Suicide. British Medical Bulletin, 100, 101-121. doi:10.1093/bmb/ldr042

Wittchen, H.U., Jacobi, F., Rehm, J., Gustavsson, M., Jonsson, B., Olesen, J., ... Steinhausen, H.C. (2011). The size and burden of mentaldisorders and other disorders of the brain in Europe 2010. EurNeuropsychopharmacol, 21(9), 655-679. https:// www.ncbi.nlm.nih.gov/pubmed/21896369

Whiteford, H. A., Degenhardt, L., Rehm, J., Baxter, A. J., Ferrari, A. J., Erskine. H., ... Vos, T. (2013). Global burden of disease attributable to mental and substance use disorders: Findings from the Global Burden of Disease Study 2010. Lancet, 382(9904), 1575 - 1586 doi: pii: S0140-6736(13)61611-6. 
World Federation for Mental Health.(2010). Enfermedad mental y suicidio: guía para la familia para poder enfrentar y reducir los riesgos. Woodbridge: USA. https:// consaludmental.org/publicaciones/Enfermedad-Mental-Suicidio.pdf

World Health Organization (WHO). (2014). Preventing suicide: a global imperative. Recuperado de http://www.who.int/mental health/suicide-prevention/world_report 2014/es/

Yen, S., Shea, M. T., Sanislow, C. A., Grilo, C. M., Kodol, A. E., Gunderson, J. G., ... Morey, L. C. (2004). Borderline personality disorder criteria associated with prospectively observed suicidal behavior. Am J Psychiatry, 161(7), 1296-1298. https:// www.ncbi.nlm.nih.gov/pubmed/15229066

Yoshida, K., Tonai, E., Nagai, H., Matsushima, K., Matsushita, M., Tsukada, J., ... Nishimura, R. (2006). Long-term follow-up study of borderline patients in Japan: a preliminary study. Compr Psychiatry, 47(5), 426-432. https://www.ncbi.nlm.nih.gov/ pubmed/16905408 